Session number

\title{
Passport to the Materials World: Materials Engineering Outreach Activities
}

\author{
Katherine C. Chen, Lisa Christensen, and Amanda Runciman \\ Materials Engineering Department, California Polytechnic State University, \\ San Luis Obispo, CA 93407
}

\begin{abstract}
The students and faculty of the Cal Poly State University Materials Engineering (MatE) department have put together an exciting and portable outreach program that includes an interactive presentation, demonstrations, and hands-on activities. Several of the different outreach activities will be discussed, with special emphasis on the imaginative visit to MatEland, which is accompanied with a passport for stamps at each materials demonstration station. Participants begin to view the world through the material engineer's eyes and realize the impact of materials on everyday products. In addition, new and fascinating materials, such as amorphous metal, ceramic superconductors, shape memory alloys, and instant polymeric snow are highlighted. While pedagogical techniques used in the college classroom have been applied to our outreach program, many parts of the outreach presentation have worked well at the college level also. The efforts have resulted in young children becoming excited about materials, as well as providing creative and educational opportunities for our college students. Presentation skills, knowledge of the field, and connections to the community are enhanced for the materials engineering students.
\end{abstract}

\section{Activities}

In efforts to inform young children and the general public about the field of Materials Science and Engineering, the Materials Engineering (MatE) department at Cal Poly, San Luis Obispo has been visiting local area schools and offering workshops on campus. Our program is driven by our current students and is continually evolving. We have created a set of presentation topics, demonstrations, and activities that can be easily modified for different audiences and venues.

One of our most successful events is part of the Cal Poly Society of Women's Engineers (SWE) Building an Engineer Workshop for $7^{\text {th }}$ and $8^{\text {th }}$ grade students. We create an imaginary place onn campus called "MatEland," complete with several different attractions to visit during the participants' journey. A passport is issued to every participant, and each page highlights a different attraction or activity in our labs (Figure 1). Each attraction has a creative name such as "Metropoglass" or "Planet of the Shapes." When each person visits an attraction, they received a stamp in their passport (Figure 2). Many of the attractions are popular demonstrations used by many others ${ }^{1-3}$, but here, they are just reformatted into a fun package. We have the Mayor of MatEland (a current Materials Engineering student) give a welcome speech and an introduction to the activities. A visitor book is available for them to sign and to let us know what they think of their travels to MatEland. 
Figure 1. Examples of the "attractions" in MatEland found in the passports. Visitors get a stamp in their passport after completing the activity or demonstration.

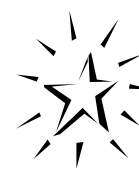
Planet of the Shapes

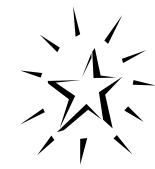

Atlantis
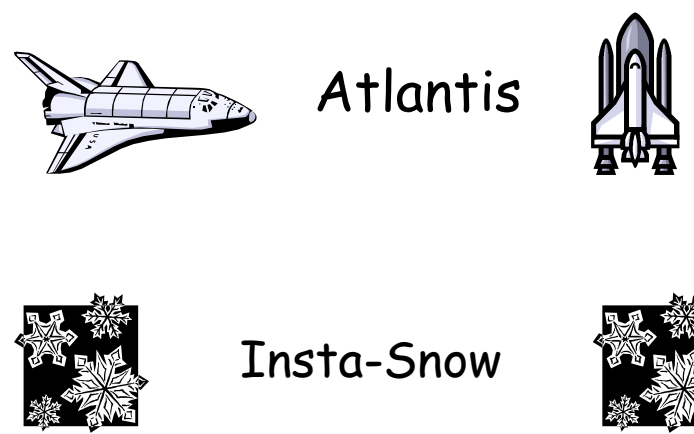

Insta-Snow

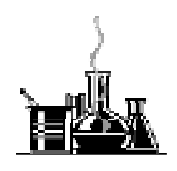

\section{Bounce}

House

\section{Charpy Town}
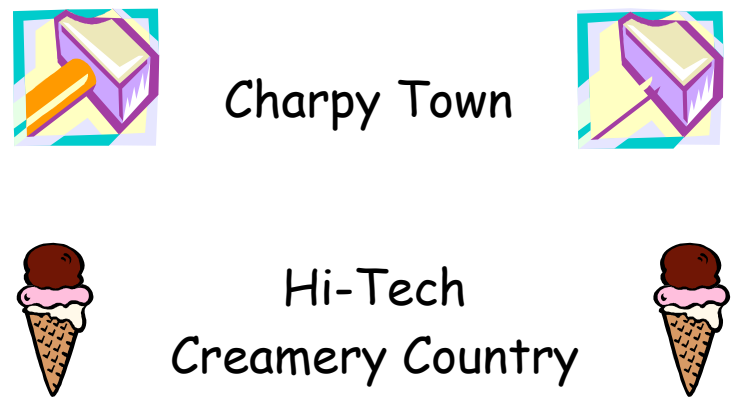

\section{Hi-Tech \\ Creamery Country}

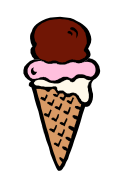

Metropoglass
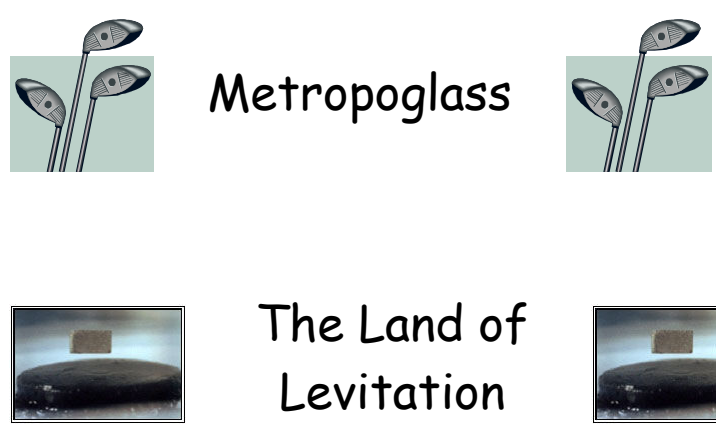

Shape Memory Alloys are an amazing group of metals that have the ability to "remember" their original shape after being deformed or changed. The most popular Shape Memory Alloy is nickel titanium, NiTi.

The space shuttle tile is very porous and thus has very good insulating properties. These tiles protect the shuttle from the high temperatures experienced during reentry from space. The insulating properties allow the tile to be handled on one side while being heated with a blow-torch on the other.

Insta-Snow is a special superabsorbent polymer powder that expands to 100 times its original size when water is added. The polymer chains of the dry powder are tightly packed around each other. Water causes the polymer chains to unravel making the powder grow into snow!

In this experiment, you use white glue and borax to make a polymer! The glue has many vinyl monomers that are not connected in long chains. Borate ions from the borax cause the monomers to link together forming long polymer chains. The properties of the glue change from sticky to stretchy when monomers link together.

The Charpy Impact test shows how much energy a material absorbs during fracture. The surfaces of the broken parts can be viewed as brittle or ductile.

Testing the same type of material at high, low, and room temperatures shows how a material changes from brittle to ductile at a transition temperature.

This experiment shows the rapid solidification of a milk and sugar mixture. The liquid nitrogen uses energy from the milk and sugar to change from a liquid to a gas. The absorption of heat by the liquid nitrogen solidifies the milk and sugar into yummy ice cream!

Metals that are processed to be noncrystalline are known as "Metallic Glass". This material allows the steel ball to "bounce" elastically because of the random orientation of the atoms. Golf clubs made of Metallic Glass drive the ball farther than those made of ordinary materials.

Superconductors used in this experiment are made from $\mathrm{YBa} 2 \mathrm{Cu} 307$. When they are cooled under a critical temperature a small magnet will float above them. The future of fast monorail transportation may lie in superconductors. 


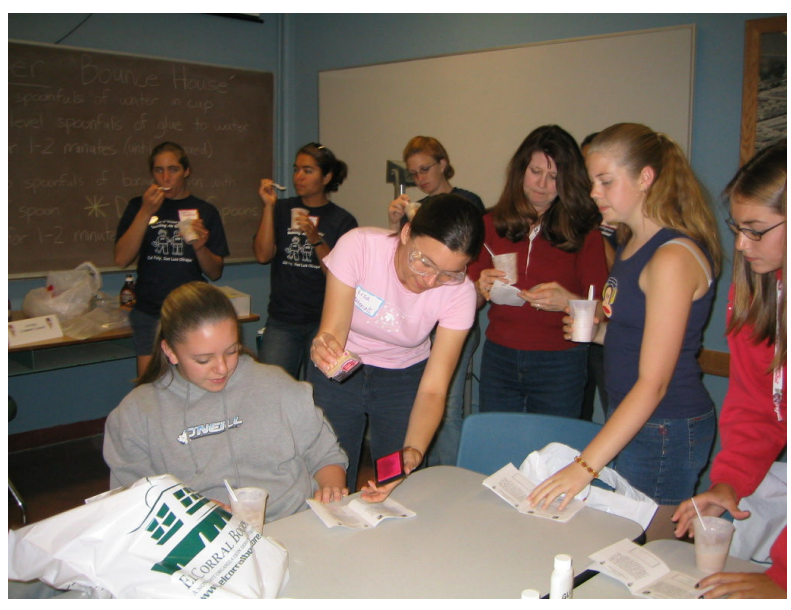

Figure 2. Student getting a stamp in her MatEland passport for Hi-Tech Creamery Country (i.e., liquid nitrogen ice cream).

The workshop runs roughly 1-1.5 hours, and several current Materials Engineering students help out. Different stations are placed throughout a large classroom, and represent the different attractions. An engineering student or volunteer is available at each attraction to give personal attention to the visitors (which may include parents). These same ideas can be applied to an open house, summer camp, workshop, etc. For classroom visits, we also have very simple PowerPoint slides with lots of pictures and very little words. Naturally, not all the activities are possible when visiting other locations.

\section{Pedagogy and Tips}

Not surprisingly, the strategies for successful outreach activities are the same approaches that are effective to the learning of college students. Our experiences in presenting difficult concepts (e.g., shape memory effects) to younger students have actually helped with our own teaching at the college level.

\section{Relating to the audience}

Our approach is basically to make things as simple and as fun as possible. We take the concepts and explain them on a simple level, and we try to relate them to activities and examples that the audience are familiar with. We spend a lot of time making sure to use appropriate vocabulary for the age group, so that we avoid talking over anyone's head. For instance, the presenters practice explaining concepts without invoking any materials science terminology, such as dislocations. It is important to not overwhelm the audience and make explanations too complicated for them to understand. Starting from the same knowledge base (such as materials are everywhere!) makes the visitors comfortable and willing to learn further.

We typically open our program by asking the visitors to think about the things around them. We might ask them about the chair they are sitting in, or the toothbrush they used this morning, or the bicycle they rode to school. We want them to realize that these are all made of different materials for a particular reason. Even though they may not have realized it until that moment, we get them to see that they know what materials are. After all, we are "living in a material world"4. By starting out with a fun and easy exercise, they quickly realize that they can relate to what we say and they listen more attentively. They begin to see how important materials are in their everyday lives. 
Once they realize that materials are everywhere, we talk to them about how materials engineers interact with materials. We explain how we try to understand why materials behave the way they $d o$, in order to decide which material to use to make an airplane, a swing set, or a cell phone. They always get excited when we tell and show them how we get to break things and make things.

\section{Using interactive, sensory activities}

The more that the audience can participate in the outreach activities, the better experience they have. Thus, for the MatEland tour, the group gets broken down into teams of 2-3 children that visit different stations at the same time, and then wander around to new stations at their own pace. Each station has a college student who supervises the activity and can give personal attention to the group. Almost every participant is able to try out the demonstrations on their own. Often times, younger students are more apt to ask questions in the small setting rather than during a large classroom presentation.

For the cases where only one presentation can be given to a large group, we make sure we bring up volunteers from the audience to perform the demonstrations. We also make sure that we have a diverse population for the volunteers (rather than always choosing the loudest and most eager person in the front). To make the presentation more interactive, we pose specific, well-prepared questions to get the audience thinking (much like giving effective lectures). We also allow ample opportunity for the audience to ask questions.

A general guiding principle for our outreach activities is to allow the participants to perform the activities themselves and discover things on their own. Thus, for NiTi shape memory alloys, we let the participants bend the wires to a different shape, and then use hair dryers or hot water to get the wires to remember their previous shapes. Multiple samples on hand are advisable.

The "hands-on" approach to learning can be applied to outreach activities as well. Anytime the participants can experience a demo using other senses, besides just watching and/or hearing, the retention of the activity is much higher. A favorite and popular demonstration of ours has turned out to be NiTi superelastic rods. As the NiTi rod is bent around a person's grip (Figure 3), the applied strain induces the solid to solid phase transformation and consequently, heat is released. The hand quickly feels an increase in temperature. As the rod is unloaded and the reverse transformation occurs, heat is taken away from the hand and the rod becomes cold. Other sensory experiences are possible with $\mathrm{NiTi}^{5}$.

Figure 3. Phase transformations can be felt as heat on the bent NiTi superelastic rods.

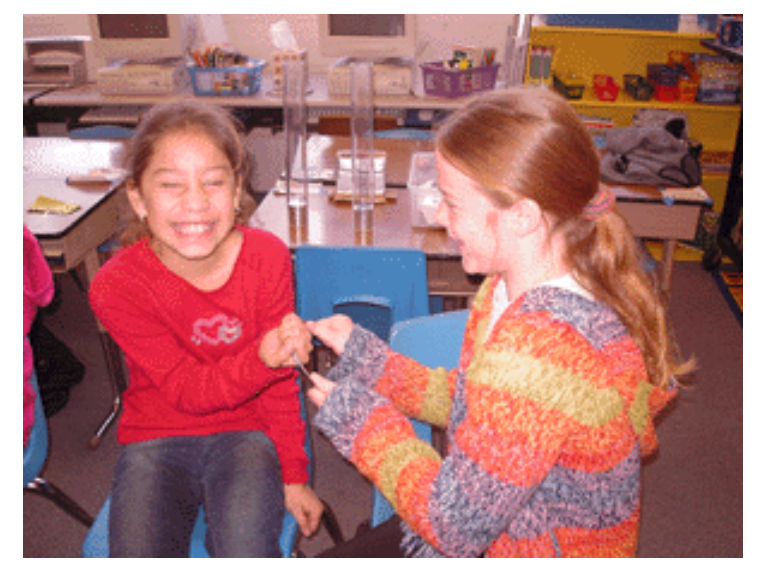




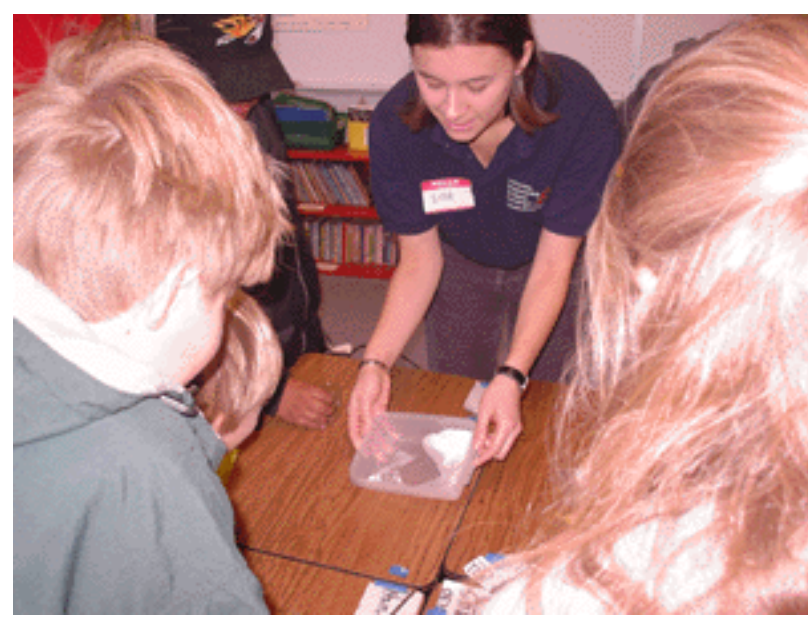

Figure 4. Instant snow results from polymer powders absorbing water and swelling. The fluffy powder feels cold, like real snow.

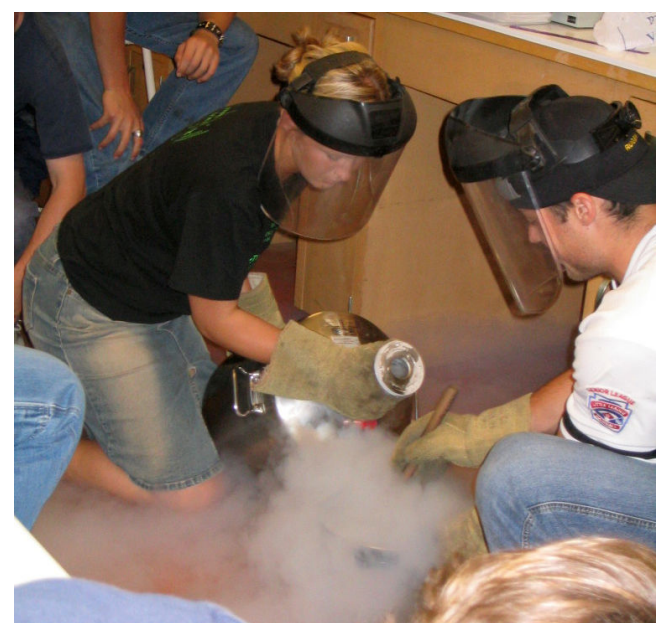

Figure 5. Rapid solidification of ice cream mixture by liquid nitrogen provides a memorable and tasty treat.

For the instant snow demonstration ${ }^{6}$, a polymer powder absorbs water and fluffs up (Figure 4), and participants are usually very eager to touch the snow. The newly made snow can also be put in a zip lock bag to be given away. Follow-up questions can be asked to predict what will happen to the snow as the moisture evaporates from the polymer with time. Models of a polymer chain that can coil and stretch are especially useful to explain the phenomenon. Many people are also intrigued by the use of the same polymer for fake snow in Hollywood movies.

While eating is usually prohibited in labs, we allow tasting of our special rapidly solidified materials - liquid nitrogen ice cream (Figure 5) ${ }^{7}$. Again, phase transformations can be discussed as the visitors eat their creation. Explanations are made as to how the liquid nitrogen turns the liquid ingredients into solid ice cream, how it produces very small ice crystals, and how it aerates the ice cream as it boils away. This is by far our most popular and tasty demonstration.

\section{Connecting to the "real world"}

While many of the demonstrations or activities have "wow" factors associated with them, it is important not to lose sight of how they demonstrate important principles in Materials Science and Engineering and to give practical applications of the materials.

Nickel titanium (NiTi) alloys are finding their way into many unique applications. Superelastic properties (e.g., strain-induced phase transformations) enable flexible eyeglass frames and many biomedical devices. Stents that prop open arteries for blood flow give a good example of how materials engineering can help people, which is an important factor when choosing majors for many women and minorities. Actual products and items to pass around are extremely effective.

Using current events or case studies are another good way to present Materials concepts. The space shuttle nicely introduces the insulating ceramic demonstration that involves a blow torch. 
Figure 6. a) Amorphous metal demonstration shows how the returned energy can be used to make b) better golf clubs. a)

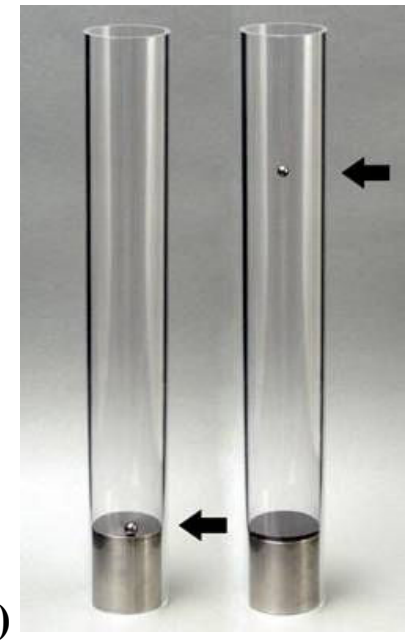

b)

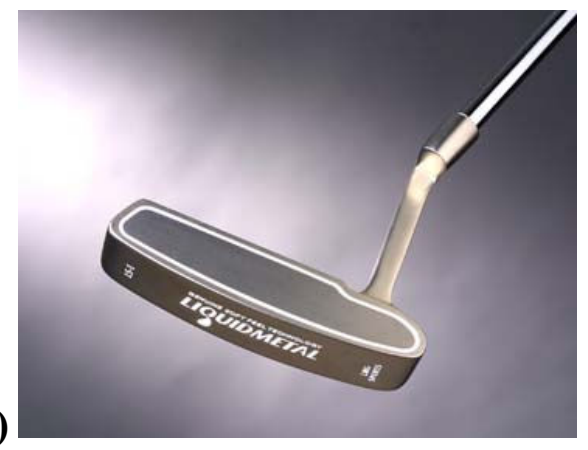

Likewise, the Titanic can be related to the Charpy Impact tests of steels at different temperatures. Students usually enjoy fire and breaking things, and thus these demonstrations are a good way to grab people's attention for learning.

Sports equipment is also an enticing way to present materials to students at all levels. In the metallic glass demonstration ${ }^{8}$, there are two pieces of metal at the bottom of a plastic tube (Figure 6a). One piece of metal is steel and the other is coated with a layer of metallic glass (or amorphous metal). Volunteers drop a small metal ball bearing in each tube at the same time and get to see how much higher and longer the ball dropped on the metallic glass bounces. Atomic arrangements and crystallinity can then be explained, and liquid metal golf clubs (Figure $6 \mathrm{~b}$ ) ${ }^{9}$ can be introduced.

\section{Involving college students}

The success of our outreach activities can be attributed to our enthusiastic Materials Engineering students. They are the ones that really enable the programs to work. Many teachers and adults comment on how effective the college students are in making science and engineering exciting to younger children (Figure 7). Each year, the engineering students play a role in creating the PowerPoint presentation so that they have an invested interest in the outreach activities. They

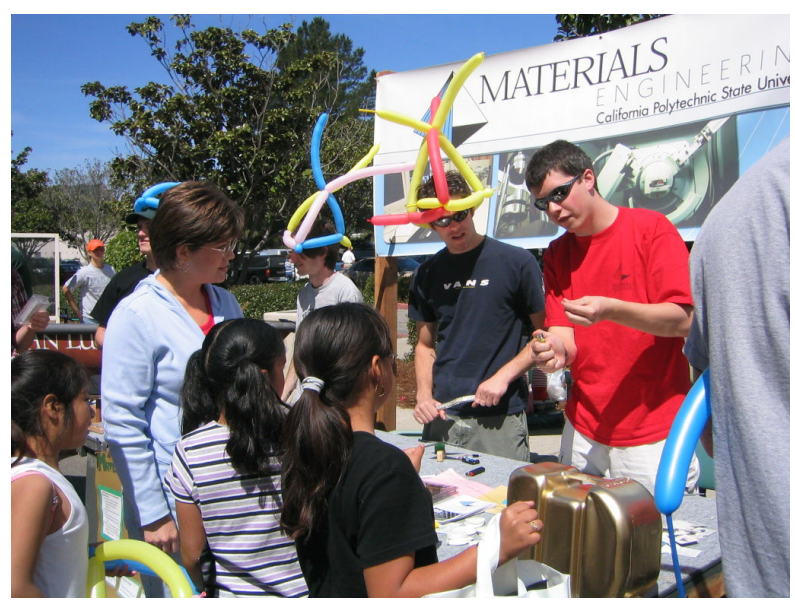
are able to be creative and also interact with the community. Many of the college students become more devoted to the department and these students tend to stay more connected after they graduate. We are now starting up an alumni outreach program where our alums can use our same demonstrations and presentation in their own communities.

Figure 7. Materials Engineering students show how materials are cool and fun to young children. 


\section{Outcomes}

While we have no formal assessment of how much our outreach participants actually learn about materials, we believe that we do make some impact and at least provide a positive, fun experience associated with science and engineering. As part of the MatEland sign-in guest book, we also asked the visitors the simple question, "What do You think about Materials Engineering?" (Figure 8). The comments were affirmative and thankful. Our main goal was to get the participants so excited about materials that they go home and share their experience with their family and friends.

On the other end, our Materials Engineering college students not only enjoy the experience, but also develop valuable presentation skills. They gain a better and deeper understanding of the materials concepts after having to teaching them to others. The students also gain an appreciation for volunteering and realize that they have something to offer others. After realizing the benefits to our students who help out with the outreach activities, we are now trying involve many more students. Often times, students that might not perform well on tests in classes turn out to be excellent outreach presenters.

While starting an outreach presentation from scratch can require a large amount of time, the rewards are plentiful. Many existing demonstrations exist in papers, books and online. Each group can find their own personal touch and have fun. Applying the same approaches of teaching lectures to giving outreach presentations can be beneficial. Likewise, many ideas and demonstrations that were developed for outreach activities have found their way into college courses. Perhaps our best measure of success is the numerous repeat requests that we get from different schools and organizations.

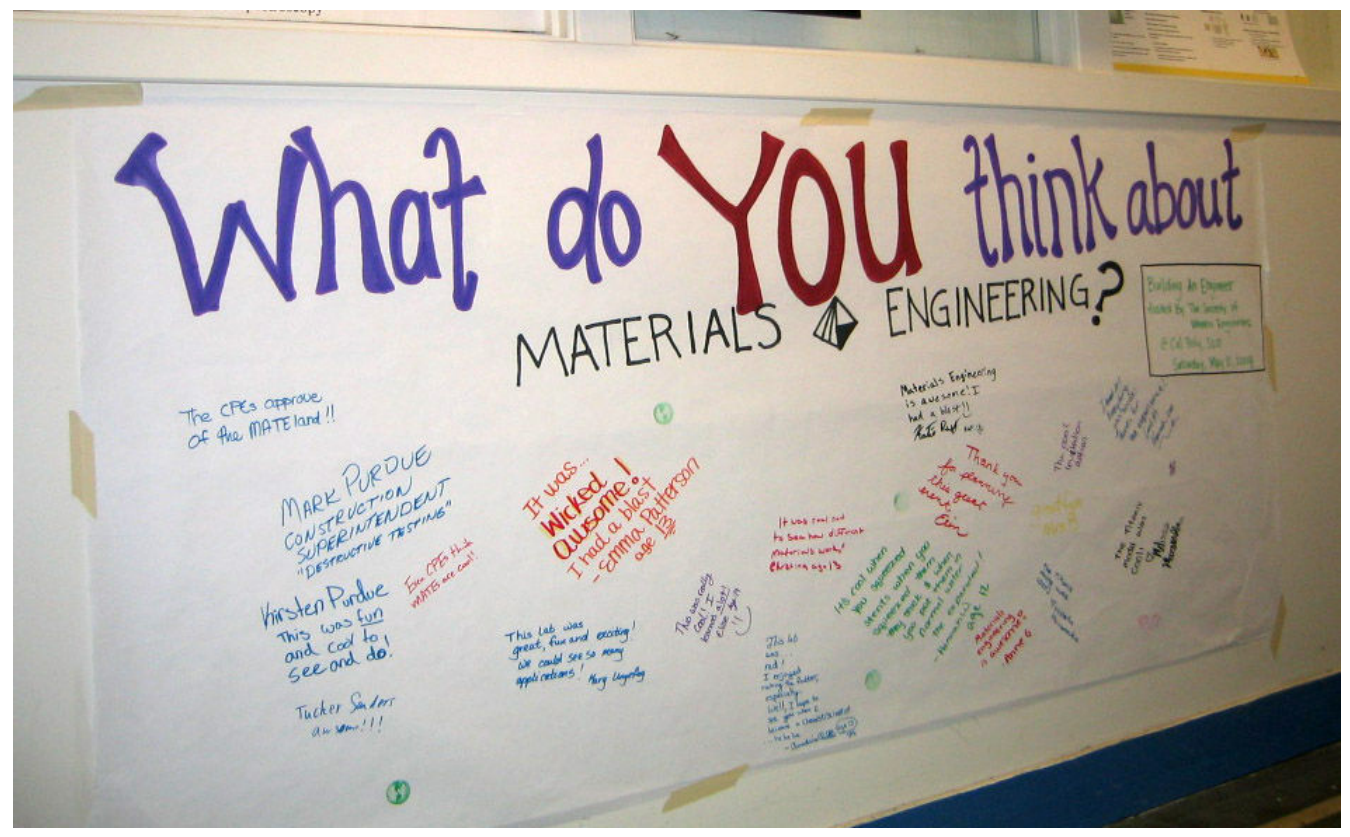

Figure 8. The MatEland visitor guest book for signatures and comments about their visit. 


\section{Bibliographic Information}

1. "Classroom Demonstrations: Suggested Classroom Demonstrations and Laboratory Experiments", Instructor's Resource CD to accompany Fundamentals of Materials Science \& Engineering 1e, William D. Callister.

2. http://mrsec.wisc.edu/edetc/takeout/index.html

3. http://mst-online.nsu.edu/new/

4. "Material Girl," Madonna, 1984.

5. K.C. Chen, W.C. Crone, and E.J. Voss' "Shape Memory Alloys for Classroom Demonstrations, Laboratories, and Student Projects," MRS Symposium Proceedings, April 2004;

http://www.mrs.org/publications/epubs/proceedings/spring2004/bb/index.html

6. http://www.stevespanglerscience.com/product/1443

7. http://www.strangematterexhibit.com/demonstrations.html

8. http://mrsec.wisc.edu/edetc/amorphous/index.html

9. http://www.liquidmetaltechnologies.com/

\section{Biographical Information}

KATHERINE C. CHEN is an Associate Professor in the Materials Engineering Department at Cal Poly State University, San Luis Obispo, CA. She received her bachelor degrees (in Chemistry and Materials Science \& Engineering) from Michigan State University, and Ph.D. from the Massachusetts Institute of Technology. At Cal Poly, she teaches undergraduate students Structures of Materials, Kinetics of Materials, and various other courses.

\section{LISA CHRISTENSEN}

Lisa Christensen graduated from California Polytechnic State University, San Luis Obispo in 2004 with an MS in Engineering, specializing in Biomedical Engineering. Her thesis research focused on understanding fracture and fatigue behavior in cortical bone. Prior to her graduate studies, she earned her BS in Materials Engineering in 2002. Currently, Lisa works as a design engineer in the biomedical industry and heads the alumni outreach program.

\section{AMANDA RUNCIMAN}

Amanda Runciman graduated with her Bachelor of Science in Materials Engineering from the California Polytechnic State University (Cal Poly), San Luis Obispo in June of 2004. She is currently pursuing her Master of Science in Materials Engineering at Cal Poly, and teaches a materials engineering lab at Cal Poly. She is also heavily involved with the department outreach program. 